Journal for

.... ImmunoTherapy of Cancer

\section{Avelumab for advanced Merkel cell carcinoma in the Netherlands: a real- world cohort}

To cite: Levy S, Aarts MJB, Eskens FALM, et al. Avelumab for advanced Merkel cell carcinoma in the Netherlands: a real-world cohort. Journal for ImmunoTherapy of Cancer 2020;8:e001076. doi:10.1136 jitc-2020-001076

Accepted 18 August 2020
Check for updates

(C) Author(s) (or their employer(s)) 2020. Re-use permitted under CC BY-NC. No commercial re-use. See rights and permissions. Published by BMJ.

For numbered affiliations see end of article.

Correspondence to

Sonja Levy; so.levy@nki.nl

\section{ABSTRACT}

Background Merkel cell carcinoma (MCC) is associated with high recurrence rates and poor survival when metastatic disease is present. The immune checkpoint inhibitor avelumab has shown high response rates (RRs) and durable responses in patients with advanced MCC (aMCC) in clinical trials. To date, only results from clinical trials, patients treated in an expanded access program and very small numbers of patients have been reported. In this study, detailed real-world efficacy and toxicity data of avelumab in patients with aMCC are reported. Methods Patients with aMCC treated in four dedicated referral centers in the Netherlands were analyzed from February 2017 until December 2019. Patients were included if they had received at least one administration of avelumab, regardless of previous lines of therapy. Patient data were collected retrospectively from patient records. Primary endpoints were response rate (RR) and duration of response (DOR). Secondary endpoints were progressionfree survival (PFS), overall survival (OS), and toxicity. Results Fifty-four patients received avelumab. Eight (15\%) patients had locally advanced disease (laMCC). In $40(74 \%)$ patients, avelumab was first-line treatment, these included all patients with laMCC. The median follow-up was 8.9 (range 0.5-35.9) months. RR was 57\% $(n=31)$ with $24 \%(n=13)$ of patients achieving a complete response. The median DOR was 8.4 (range 1.3-22.1) months and $23(43 \%)$ patients had an ongoing response at the end of the study. The median PFS was $8.6(95 \% \mathrm{Cl}$ 1.6-15.5) months, and the median OS was $25.8(95 \% \mathrm{Cl}$ 9.1-42.4) months. Six (11\%) patients experienced grade 3 toxicity. No grade $4-5$ toxicity was seen.

Conclusions In this real-world cohort, clinical efficacy and toxicity outcomes in clinical practice were in line with results from clinical trials and showed relatively high RRs and durable responses in patients with aMCC.

\section{INTRODUCTION}

Merkel cell carcinoma (MCC) is a rare and potentially aggressive neuroendocrine carcinoma of the skin with an incidence of around $0.5-0.8 / 100000 .^{1-4}$ Incidence has been rising over the last few decades. ${ }^{2}{ }^{3}$ This is thought to be due to not only improved diagnostics and better awareness of this illness, but also increasing sun exposure and an aging population. The median age of presentation is 75 years and in approximately $7 \%-27 \%$ of patients regional or distant metastases are present at diagnosis. ${ }^{12}$ Prior to the introduction of immunotherapy for this disease, patients who no longer had curative, surgical treatment options due to metastatic MCC (mMCC) or locally advanced MCC (laMCC), had 5-year overall survival (OS) rates of only $7 \%-12 \% .^{56}$ Treatment strategies for advanced MCC (aMCC), including both laMCC and mMCC, were historically based on those for other small cell malignancies, such as small cell lung cancer, and mostly consisted of polychemotherapy. Although initial response rates (RRs) of aMCC to platinum-based chemotherapy were high, patients rapidly relapsed and no durable responses or survival benefit have been reported. ${ }^{17}$

MCC is associated with two different pathways of pathogenesis. The first route involves the Merkel cell polyomavirus (MCV). MCV is present in up to $80 \%$ of patients with MCC in the Northern hemisphere and integrates into the genome of cells driving oncogenic processes such as expression of T-antigen oncoproteins. ${ }^{8-11}$ In MCV-negative MCC, exposure to ultraviolet (UV) radiation appears important in the pathogenesis. MCV-negative tumors mostly arise from sunexposed areas of the skin and show a high mutational burden and adaptive immune responses that are associated with chronic exposure to UV radiation. ${ }^{12} 13$ These alternative pathways of pathogenesis both provide a good rationale for treatment with immune checkpoint inhibitors (ICI). ${ }^{14} 15$

Several clinical trials showed beneficial results of ICI, such as programmed cell death-1/programmed cell death ligand-1 (PD-(L)1) inhibitors, in the treatment of aMCC. ${ }^{8} 1617$ Pembrolizumab was shown to have an objective RR (ORR) of $56 \%$, with 
progression-free survival (PFS) at 6 months of $67 \%$ in 26 patients with aMCC. ${ }^{17}$

In 2016, the JAVELIN study, a phase II clinical trial that investigated avelumab treatment in patients with aMCC, showed significant and durable responses. ${ }^{8}$ In this study, 88 patients who had progressed after chemotherapy were treated with avelumab and an ORR of $31.8 \%$ was seen, with 8 patients achieving a complete response (CR) and 20 patients a partial response (PR). The median follow-up was 10.4 months. Based on this study, patients in the Netherlands were able to receive avelumab within an expanded access program (EAP). Avelumab was granted accelerated approval for aMCC by the Food and Drug Administration in North America in March 2017, which was followed by the European Medicines Agency in September of 2017. In November of 2017, reimbursement in the Netherlands followed and avelumab was integrated into the routine management of patients with aMCC. ${ }^{18}$

The population of patients with aMCC is frequently elderly and frail, making it essential to determine whether the results described in a clinical trial population can be replicated in a real-world setting. In 2019, Knepper et al performed a large genomic analysis of patients with MCC, and investigated the response to various ICI in 36 patients with aMCC, of which 10 were treated with avelumab. There, a RR of $44 \%$ was seen in all 36 patients. ${ }^{13}$ More recently, a large study was performed in an elegant attempt to evaluate the clinical efficacy and safety of avelumab in the real-world population. There, the authors included patients with aMCC who had received avelumab in the EAP. They found that ORR was $47 \%$, with $23 \%$ of patients achieving a CR. Unfortunately, although a large number of patients were evaluable for response $(n=240$, $46 \%$ of total), data were limited because the evaluation of progression and toxicity were not documented according to a study or clinical protocol, but was at the discretion of the treating physician to document in the EAP system. Also, the duration of response (DOR) was merely based on the resupply of avelumab and data on the medical history of patients included were sparse. ${ }^{19}$

Both the clinical trials and the results from the EAP indicate an auspicious effect of avelumab in treatment with aMCC, but detailed data on patients with aMCC treated with avelumab in routine clinical practice are still lacking.

In the Netherlands, patients with aMCC are treated in four dedicated tertiary referral centers across the country. In this nationwide study, we aimed to evaluate the efficacy and toxicity of avelumab in a large real-world cohort of patients with aMCC treated in routine clinical practice in the Netherlands.

\section{METHODS \\ Patients}

Patients with aMCC treated with avelumab since the introduction of the EAP in the Netherlands were included from all four MCG referral centers from February 2017 until December 2019. Data were collected retrospectively and patients were followed-up until death or end of follow-up. Patients were excluded if they had received other types of ICI prior to avelumab. Histopathological analyses were performed during the diagnostic workup according to the standard of care for these tumors. MCV positivity was determined immunohistochemically using a CM2B4 monoclonal antibody as described previously. ${ }^{2021}$

Avelumab was administered in a 2 weekly interval as per institutional protocol. Premedication consisting of $2 \mathrm{mg}$ clemastine and $1000 \mathrm{mg}$ paracetamol was administered intravenously during the first three cycles and continued thereafter only if infusion reactions occurred.

Patient characteristics, response to avelumab, adverse effects, and toxicity were gathered from electronic patient records. All patients gave consent to use their medical data according to institutional protocols.

\section{Outcomes}

Primary endpoints were RR and DOR. Response evaluation by CT or positron emission tomography (PET-)CT was performed at approximately 12-week intervals. As this study was not conducted within a trial setting, the response was reported in radiological records according to routine diagnostic practice. When the radiological evaluation was not possible, clinical parameters such as changes in visible skin lesions that were measured with a caliper or other evaluable parameters such as performance status were used. For biochemical response measurements, all centers used lactate dehydrogenase (LDH) with an upper limit of normal (ULN) $248 \mathrm{U} / \mathrm{L}$, and additionally, neuron-specific enolase (NSE) with a ULN $18.2 \mu \mathrm{g} / \mathrm{L}$ was used in the largest referral center. ${ }^{22}$ Responses were extracted retrospectively from patient records and radiology reports. The measurements in the reports initially described by a radiologist were reassessed according to RECIST criteria. PR was defined as radiographic shrinkage of tumors $\geq 30 \%$. In the absence of radiological response evaluation, visible and/or palpable shrinkage $\geq 30 \%$ of skin tumors and/or lymph nodes were evaluated. CR was defined as a complete metabolic and radiological response on PET-CT. When mixed response (MR) was present at $\geq 2$ consecutive response evaluations, defined as $\geq 30 \%$ tumor shrinkage, with simultaneous growth $\geq 20 \%$ of other lesions and/or occurrence of new lesions, a consensus on continuation or cessation of avelumab was reached in a multidisciplinary team (MDT). The decision to perform salvage treatment including surgery or radiotherapy was also reached in an MDT. Progressive disease (PD) was defined as radiographic tumor growth $\geq 20 \%$ and/or growth of visible skin tumors, and/or increase of biochemical markers such as LDH and/or NSE above the ULN, and/or deterioration of a patients' performance status due to aMCC. DOR was defined as the time from first documented PR or CR until documented PD, death, or end of follow-up.

Secondary endpoints were PFS, OS and toxicity. PFS and OS were defined as the time from the first administration 
of avelumab until documented progression or death, respectively. Patients were censored at the end of the study. Toxicity was evaluated according to CTCAE V.5.0, grades $1-5$ were included.

\section{Statistical analysis}

Descriptive statistics with median and ranges were used for continuous variables and frequency and percentages for categorical variables. Pearson's $\chi^{2}$ test was used to compare responses between groups and for the forest plot, univariable Clopper-Pearson calculations were performed to establish confidence intervals for proportions of patients that responded to avelumab. The KaplanMeier method was used to evaluate OS and PFS and the log-rank test was performed for comparison between firstline and second-line treatment. IBM SPSS V.25 was used to perform all statistical analyses.

\section{RESULTS}

\section{Baseline characteristics}

We identified 55 patients with aMCC who had received at least one dose of avelumab, 54 of these patients fulfilled the inclusion criteria. One patient was excluded due to prior treatment with ICI (nivolumab). Two patients received avelumab in the EAP. The first administration of avelumab was in February 2017, the last patient started treatment in September 2019.

Patients were first diagnosed with MCC at a median age of 71 (range 50-86) and had a median age of 73 (range 53-88) years at the start of avelumab. Thirty-four $(63 \%)$ patients were male individuals. Primary tumor localizations were head and neck, trunk, extremities, or unknown primary tumor (UPMCC) in 13 (24\%), 8 $(15 \%), 25(46 \%)$, and $8(15 \%)$ patients, respectively. Eight $(15 \%)$ patients had locally advanced (stage IIIB/ laMCC) disease and $46(85 \%)$ had the distant disease (stage IV/mMCC) at the start of avelumab administration. Of the latter, $35(65 \%)$ patients had distant nodal and/or (sub)cutaneous disease, and in 19 (30\%), visceral and/or peritoneal/mesenterial metastasis were present. In $12(22 \%)$ patients, one organ site was involved, and in $4(7 \%)$ patients, disease was present in two organs. Seven (13\%) patients had a history of immunosuppression, including chronic lymphatic leukemia, Waldenström's macroglobulinemia, human deficiency virus, idiopathic pulmonary fibrosis, and a kidney transplant. MCV status was determined in 21 patients. Of these, 15 $(71 \%)$ was positive. PD-(L) 1 expression was not determined in routine clinical practice, hence no data on PD-(L) 1 expression were available. LDH levels were available for 50 patients at the start of avelumab. Of these, 29 (58\%) had elevated LDH levels above ULN. Baseline characteristics are shown in table 1 . Avelumab was firstline treatment for all patients with laMCC $(n=8,15 \%)$ and in 32 (59\%) patients with mMCC; the remaining 14 (26\%) patients with mMCC received avelumab as secondline treatment. Prior therapy in all patients consisted of
Table 1 Baseline characteristics for all included patients

\begin{tabular}{|c|c|}
\hline Characteristics & All patients $(\mathrm{N}=54)$ \\
\hline \multicolumn{2}{|l|}{ Sex, n (\%) } \\
\hline Male & $34(63)$ \\
\hline Female & $20(37)$ \\
\hline \multicolumn{2}{|l|}{ Immunosuppression history, n (\%) } \\
\hline CLL & $3(6)$ \\
\hline WM & $1(2)$ \\
\hline HIV & $1(2)$ \\
\hline IPF & $1(2)$ \\
\hline KT recipient & $1(2)$ \\
\hline \multicolumn{2}{|l|}{ Age (years), median (range) } \\
\hline At diagnosis & $71.1(50.2-86.3)$ \\
\hline At start IT & $73.0(53.0-88.0)$ \\
\hline \multicolumn{2}{|l|}{ Primary tumor site, n (\%) } \\
\hline Head and neck & $13(24)$ \\
\hline Trunk & $8(15)$ \\
\hline Extremity & $25(46)$ \\
\hline Unknown primary & $8(15)$ \\
\hline \multicolumn{2}{|l|}{ WHO performance status, $\mathrm{n}(\%)$} \\
\hline 0 & $17(32)$ \\
\hline 1 & $32(59)$ \\
\hline 2 & $5(9)$ \\
\hline \multicolumn{2}{|l|}{ Disease status, n (\%) } \\
\hline Locally advanced & $8(15)$ \\
\hline Distant disease & $46(85)$ \\
\hline \multicolumn{2}{|l|}{ Metastasis, n (\%) } \\
\hline Visceral metastases* & $19(35)$ \\
\hline Nodal or (sub)cutaneous metastases & $35(65)$ \\
\hline \multicolumn{2}{|l|}{ No. of organ sites involved, $\uparrow \mathrm{n}(\%)$} \\
\hline 1 & $12(22)$ \\
\hline 2 & $4(8)$ \\
\hline \multicolumn{2}{|l|}{ Line of therapy, n (\%) } \\
\hline First & $40(74)$ \\
\hline Second & $14(26)$ \\
\hline \multicolumn{2}{|l|}{ Radiotherapy } \\
\hline Yes & $31(57)$ \\
\hline$\underline{\text { No }}$ & $23(43)$ \\
\hline \multicolumn{2}{|l|}{ MCV, n (\%) } \\
\hline Yes & 15/21 (71) \\
\hline No & 6/21 (29) \\
\hline \multicolumn{2}{|l|}{ LDH, n (\%) } \\
\hline Yes & $21 / 50$ (39) \\
\hline No & $29 / 50(54)$ \\
\hline Missing & $4 / 50(7)$ \\
\hline
\end{tabular}

*Also including distant mesenterial or peritoneal metastasis. †Organ sites included liver, bone, adrenal cortex, pancreas, intestine, pleura.

CLL, chronic lymphatic leukemia; IPF, idiopathic pulmonary fibrosis; IT, immunotherapy; KT, kidney transplant; LDH, lactate dehydrogenase; MCV, Merkel cell polyomavirus; WM, Waldenström's macroglobulinemia. 


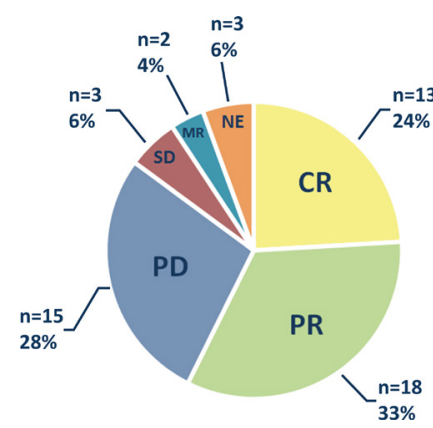

Figure 1 Best overall response during follow-up for all 54 patients. CR, complete response; MR, mixed response; NE, not evaluable; PR, partial response; PD, progressive disease; $\mathrm{SD}$, stable disease.

platinum-based chemotherapy. Additionally, 31 (57\%) patients had received radiotherapy on either primary tumor area or metastasis prior to avelumab initiation. All patients had a performance score (PS) $\leq 2$. Patients with a performance score of $2(n=5,9 \%)$ were all in the mMCC group. In patients with laMCC, $3(38 \%)$ had PS 0 and 5 (63\%) had PS 1, compared with $14(30 \%)$ and $27(58 \%)$ patients with mMCC, respectively. Patients received a median of 10 (range 1-39) doses of avelumab and the median follow-up time was 8.9 (range 0.5-35.9) months.

\section{Response to avelumab}

Three patients $(6 \%)$ were not evaluable for response: two died due to comorbidities before response evaluation. Comorbidities included rapidly progressive dementia and a superinfection due to pre-existent idiopathic pulmonary fibrosis shortly after avelumab administration. One patient discontinued avelumab after one infusion due to an allergic reaction and was referred to the general practitioner for palliative care.

Out of all 54 patients, objective response to avelumab was seen in $57 \%(n=31)$. In $24 \%(n=13)$ of patients, best overall response (BOR) was a CR, PR was seen in $33 \%$ $(n=18)$, stable disease in $6 \%(n=3)$, MR in $4 \%(n=2)$, and PD in 28\% ( $n=15)$ of patients. BORs are shown in figure 1. Response to avelumab therapy in all patients divided and analyzed by subgroups are shown in figure 2 . In patients with laMCC, RR was $50 \%(\mathrm{n}=4)$, compared with $59 \%$ $(\mathrm{n}=27)$ in patients with mMCC $(\mathrm{p}=0.646)$. Regarding CRs: in patients with laMCC $25 \%(\mathrm{n}=2)$ achieved a CR, and in patients with mMCC, $24 \%(\mathrm{n}=11)$ patients achieved a CR $(\mathrm{p}=0.947)$. In five $(9 \%)$ patients, salvage surgery or radiotherapy was performed for either residual tumor lesions $(n=3)$ or new solitary lesions $(n=2)$. All salvage interventions were in patients with mMCC. RR was $41 \%(\mathrm{n}=7)$, $63 \%(\mathrm{n}=20)$, and $80 \%(\mathrm{n}=4)$ in patients with PS 0,1 , and 2 , respectively $(\mathrm{p}=0.200)$. Of these, $\mathrm{CR}$ was achieved in $12 \%(\mathrm{n}=2), 28 \%(\mathrm{n}=9)$, and $40 \%(\mathrm{n}=2)$, respectively $(\mathrm{p}=0.303)$.

In patients with UPMCC, $63 \%(\mathrm{n}=5)$ had an objective response, whereas in patients with known primary locations $56 \%(\mathrm{n}=26)$ had a response $(\mathrm{p}=0.752)$. CRs occurred in $25 \%(\mathrm{n}=2)$ of patients with UPMCC, and

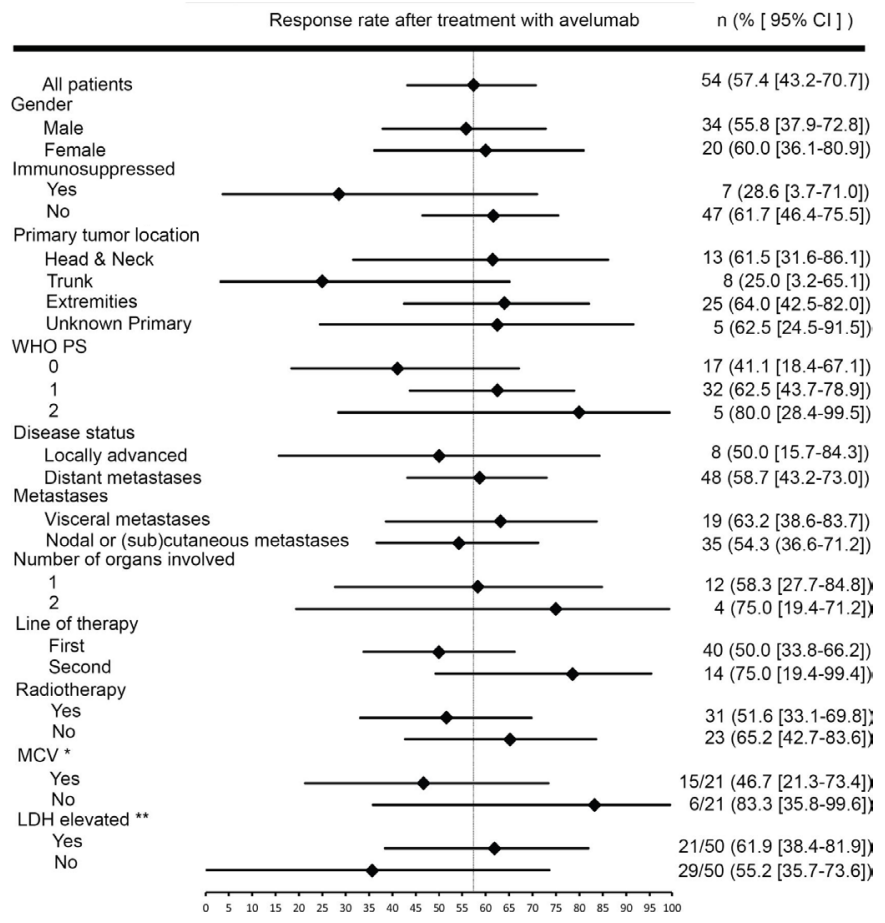

Figure 2 Response rates by subgroups. *Response rates calculated for patient with known viral status. ${ }^{*}$ Response rates calculated for patient with known LDH levels at the start of avelumab. LDH, lactate dehydrogenase; MCV, Merkel cell polyomavirus; WHO PS, World Health Organization performance status.

in $24 \%(\mathrm{n}=11)$ of patients with known primary tumors $(\mathrm{p}=0.947)$.

Out of the 19 patients with visceral metastases (including peritoneal or mesenteric metastases), 63\% $(n=12)$ had a response compared with $54 \%(n=19)$ out of the remaining 35 patients who had nodal or subcutaneous metastases only $(p=0.529)$. CRs were achieved in $37 \%(\mathrm{n}=7)$ of patients with visceral metastases and in $17 \%$ $(\mathrm{n}=6)$ of patients without visceral metastases $(\mathrm{p}=0.106)$.

In the 50 patients for whom LDH levels were known, no differences in response to avelumab between elevated LDH levels and normal LDH levels were seen. In patients with elevated LDH levels, response was seen in $62 \%$ $(\mathrm{n}=13)$ of patients compared with $55 \%(\mathrm{n}=16)$ of patients who had normal LDH levels $(\mathrm{p}=0.634)$. Interestingly, in patients who had received avelumab as second-line treatment, 11 of $14(79 \%)$ had a response compared with patients who received avelumab as first-line treatment, of which 20 of $40(50 \%)$ patients had a response (0.063). In contrast, CRs were present in $28 \%(\mathrm{n}=11)$ of patients receiving the first-line avelumab, compared with $14 \%(\mathrm{n}=2)$ of patients treated with second-line avelumab $(p=0.302)$. We saw no difference in response to avelumab for patients who had received radiotherapy prior to avelumab initiation: of the 31 patients who underwent radiotherapy, $52 \%(\mathrm{n}=16)$ had a response compared with $65 \%(n=15)$ out of 23 patients who did not $(\mathrm{p}=0.317)$.

In patients with a history of immunosuppression, $29 \%$ $(\mathrm{n}=2)$ had a PR, and no CRs occurred in this group. 

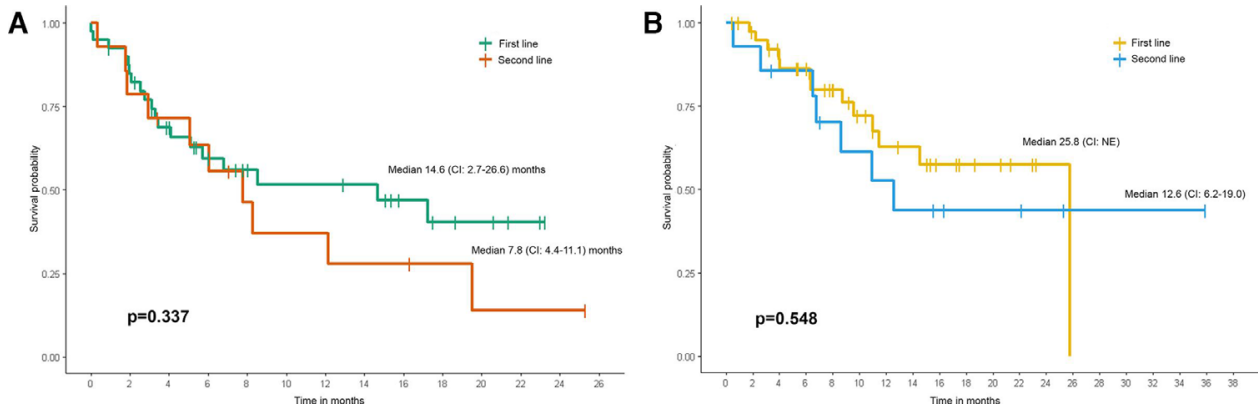

Figure 3 (A) Progression free survival all patients, divided by subgroups of patients receiving avelumab in first-line and second-line treatment. Median with $\mathrm{Cl}$ for subgroups. Subgroup comparison with $\mathrm{p}$ value by the log-rank test. (B) Overall survival of all patients, divided by subgroups of patients receiving avelumab in first-line and second-line treatment. Median with $\mathrm{Cl}$ for subgroups. Subgroups comparison with $\mathrm{p}$ value by the log-rank test. NE, not estimable.

The median DOR was 8.4 (range 1.3-22.1) months, and median PFS was 8.6 (95\% CI 1.6-15.5) months. The estimated median OS was 25.8 (95\% CI 9.1-42.4) months. We saw no significant differences in PFS and OS between patients treated with avelumab in first-line or second-line setting, $\mathrm{p}=0.337$ and $\mathrm{p}=0.548$, respectively. PFS and OS are shown in figure 3A,B. Responses were ongoing in 23 (43\%) patients at the end of follow-up. PD occurred in 7 of 19 patients for whom BOR was PR. All seven patients were on active therapy at the time of progression. For patients who achieved CR: at the end of the study, 12 of 13 patients remained free of disease, with a median DOR of 12.8 (range 3.6-22.1) months. The remaining patient had achieved a CR after 2.6 months and had discontinued avelumab treatment after 19 cycles (8.8 months). Recurrence occurred 17.2 months after the start of therapy. During follow-up, no rechallenges with avelumab were initiated for patients who had initially responded but then progressed. Clinical activity of avelumab in all patients evaluable for response are shown in figure 4 .

Out of all 54 patients, $6(11 \%)$ experienced grade 1 toxicity, which was mostly fatigue. Four $(7 \%)$ patients experienced grade 2 toxicity, which constituted of allergic reactions requiring oral intervention, hypothyroidism, and hepatitis. Grade 3 toxicity including allergic reactions

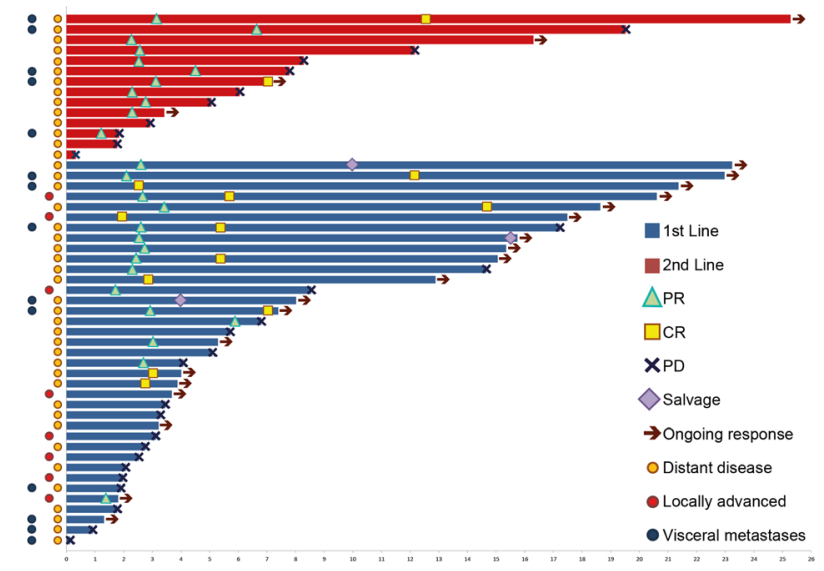

Figure 4 Clinical activity of avelumab in all evaluable patients $(n=51)$. CR, complete response; PD, progressive disease; PR, partial response. and renal insufficiency resulting in clinical admission were seen in five (9\%) patients. No grade 4 or 5 toxicities were seen. Toxicities are shown in table 2.

\section{DISCUSSION}

In this real-world cohort of patients with aMCC, avelumab treatment resulted in high RRs, durable responses, and manageable toxicities. This study describes a detailed and relatively large cohort of patients with patients with aMCC outside the setting of a clinical trial or an EAP, showing a true representation of the real-world clinical practice for patients with aMCC. We demonstrate that avelumab now has a prominent role in both first-line and second-line treatment for aMCC.

In the 40 patients in our study who received avelumab in the first-line setting, we found a RR of $50 \%$ with $28 \%$ achieving a CR. The EAP study found similar results in 15 patients who were treated with the first-line avelumab, where $47 \%$ of patients had a response. ${ }^{19}$ This is also in concordance with results seen in clinical trials involving

\begin{tabular}{|c|c|}
\hline Toxicities & Patients, n (\%) \\
\hline \multicolumn{2}{|l|}{ Grade 1} \\
\hline Fatigue & 5 (9) \\
\hline Hypothyroidism & $1(2)$ \\
\hline \multicolumn{2}{|l|}{ Grade 2} \\
\hline Hepatitis & $1(2)$ \\
\hline Allergic/infusion reaction & $1(2)$ \\
\hline Hypothyroidism & $2(4)$ \\
\hline \multicolumn{2}{|l|}{ Grade 3} \\
\hline Allergic & $3(6)$ \\
\hline Nausea/vomiting & $1(2)$ \\
\hline Renal insufficiency & $1(2)$ \\
\hline Grade 4 & $0(0)$ \\
\hline Grade 5 & $0(0)$ \\
\hline Total & $15(28)$ \\
\hline
\end{tabular}


pembrolizumab or avelumab as first-line treatment for aMCC. ${ }^{172324}$

Regarding previous lines of therapy, in the cohort of 36 patients treated with various ICIs that were investigated by Knepper $e t a l$, a dramatic decrease in response was seen in patients treated with higher therapy lines. There, a RR of $75 \%$ was found for patient treated with ICI as first-line therapy, $39 \%$ as second-line therapy, and $18 \%$ in third-line or higher-line therapy. ${ }^{13}$ Similarly, in our cohort, patients treated with first-line avelumab also had a more favorable outcome. We found that nearly all CRs arose in patients treated with the first-line avelumab, and responses were similar in both laMCC and mMCC, indicating that tumors in patients without prior lines of chemotherapy might be more sensitive to PD-(L) 1 blockade. This supports the clinical practice that we see evolving in the Netherlands in which avelumab is increasingly being used as first-line therapy for mMCC.

Although only 14 patients in our cohort were treated with avelumab as second-line therapy for aMCC, still interesting results were seen. When comparing our patients receiving avelumab as a second-line treatment for aMCC with those from clinical trials, we saw similar rates of CRs: $14 \%$ in our cohort compared with $9 \%$ in the JAVELIN trial. ${ }^{8}$ In contrast, the overall ORRs were quite different, with $79 \%$ in our second-line patients compared with $32 \%$ in the trial. There, over half of the patients were treated with more than one prior line of chemotherapy, and the authors suggest that this could have led to more immunologically depleted patients, resulting in worse RRs. This is substantiated by the updated results of the JAVELIN trial after a median follow-up of 41 months, where a trend towards a higher ORR in patients with fewer prior lines of therapy was seen. ${ }^{24}$ This could explain the higher RR in our cohort as we had no patients with more than one prior line of therapy.

In earlier years, when polychemotherapy was the treatment of choice for aMCC, a more advanced disease stage was associated with a worse prognosis. Several epidemiological studies have found that a more advanced disease stage is an independent predictor for survival. ${ }^{125-30}$ Interestingly, in studies where patients with aMCC were treated with ICI, no significant difference in response to treatment were seen between stage IIIb or IV disease, but a trend towards lower RRs remained. ${ }^{817}$ In our cohort, $35 \%$ of patients had visceral metastases at treatment initiation. This is a smaller percentage than was shown in the clinical trials, were visceral metastases have been reported to be present in $53 \%-67 \%$ of patients. ${ }^{816}$ However, older studies that focused on chemotherapy for aMCC, found similar or even lower percentages of visceral metastases than in our cohort, suggesting that the percentage of visceral metastases varies greatly between different study populations. ${ }^{731}$ Besides this, although we saw no statistically different responses, we saw a trend towards higher RRs in patients with visceral metastases. This seems contradictory to the results from clinical trials. ${ }^{82}$ Finding these differences may be attributable to the small number of patients with visceral metastases $(\mathrm{n}=19)$. Another explanation might be that because of the retrospective nature of this study, we did not perform other measurements of disease burden, such as sum of lesion diameter parameters. This might underestimate the disease burden in patients with nodal and/or subcutaneous disease only, subsequently overestimating disease burden in patients with visceral metastases. Nevertheless, our cohort represents an accurate representation of radiological documentation and response to avelumab in patients with aMCC in routine clinical practices. Therefore, these results remain generalizable to clinical practices elsewhere.

Patients with UPMCC have been shown to have a longer OS and a higher tumor mutational load than patients with known primary tumor locations. ${ }^{334}$ It has, therefore, been implied that patients with UPMCC are more likely to respond to immunotherapy. ${ }^{33}$ However, both clinical trials involving ICI and this real-world cohort did not show different RRs in patients with a UPMCC. ${ }^{8}$ Potential differences may have been missed due to the fact that we had only eight $(15 \%)$ patients with UPMCC. ${ }^{8}$ Yet our findings are in concordance with results shown in a Dutch cohort of 351 patients, where UPMCC was not associated with a survival benefit in multivariable analysis. ${ }^{35}$

As we did not perform additional genomic sequencing, data on mutational burden were not available. To determine whether the mutational burden indeed plays a role in the response, larger studies are needed. Recently, several potential strategies to involve and study larger numbers of patients with aMCC have been described. ${ }^{36}$ Future studies should aim to elucidate which patients would benefit from ICI by investigating which possible biomarkers or genomic characteristics of the tumor are associated with response to PD-(L) 1 blockade.

\section{CONCLUSION}

In the Netherlands, avelumab is increasingly being used as first-line and second-line systemic treatment for aMCC. In this real-world cohort, RRs, DOR, PFS, and toxicity results are promising and essentially in line with results found in clinical trials. Although higher RRs were seen in patients treated in the second line, more complete and durable responses were seen in patients who received avelumab as first-line treatment.

\section{Author affiliations}

${ }^{1}$ Department of Medical Oncology, Antoni van Leeuwenhoek Netherlands Cancer Institute, Amsterdam, The Netherlands

${ }^{2}$ Department of Medical Oncology, Maastricht University Medical Center+, Maastricht, Limburg, The Netherlands

${ }^{3}$ Department of Medical Oncology, Erasmus Medical Center, Rotterdam, ZuidHolland, The Netherlands

${ }^{4}$ Department of Surgery, Maastricht University Medical Center+, Maastricht, Limburg, The Netherlands

${ }^{5}$ Department of Surgical Oncology, University Medical Center Groningen, Groningen, The Netherlands

${ }^{6}$ Department of General Surgery, Erasmus Medical Center, Rotterdam, Zuid-Holland, The Netherlands 
${ }^{7}$ Department of Surgical Oncology, Netherlands Cancer Institute, Amsterdam, The Netherlands

${ }^{8}$ Department of Medical Oncology, University Medical Center Groningen, Groningen, The Netherlands

Acknowledgements The authors thank all the patients and their families, the investigators of the study and supporting teams at each of the participating centers.

Contributors METT, AvA, FALME, MJ, and MJBA: conception and design. SL: data collection and assembly; manuscript preparations. SL, MA, FE, AA, MJ, and MT: data analysis and interpretation. MT: principal investigator. All authors: manuscript editing; final approval of manuscript; accountable for all aspects of manuscript.

Funding This work was performed independently and the authors received no specific funding from governmental of commercial institutions.

Competing interests MJBA, FALME, KBMIK, LBB, DG, AvA, MJ and METT report to hold a position on an advisory board for Merck.

\section{Patient consent for publication Not required.}

Ethics approval This study was conducted in accordance with the Declaration of Helsinki and the International Council for Harmonisation Good Clinical Practice Guidelines. All patients gave consent for the use of their data.

Provenance and peer review Not commissioned; externally peer reviewed.

Data availability statement Data are available upon reasonable request. All data relevant to the study are included in the article or uploaded as supplementary information. All data are available in a deidentified data registry. Please contact the corresponding author for more information.

Open access This is an open access article distributed in accordance with the Creative Commons Attribution Non Commercial (CC BY-NC 4.0) license, which permits others to distribute, remix, adapt, build upon this work non-commercially, and license their derivative works on different terms, provided the original work is properly cited, appropriate credit is given, any changes made indicated, and the use is non-commercial. See http://creativecommons.org/licenses/by-nc/4.0/.

\section{ORCID iD}

Sonja Levy http://orcid.org/0000-0001-9457-2074

\section{REFERENCES}

1 Schadendorf D, Lebbé C, Zur Hausen A, et al. Merkel cell carcinoma: epidemiology, prognosis, therapy and unmet medical needs. Eur $J$ Cancer 2017;71:53-69.

2 Fondain M, Du Thanh A, Bessaoud F, et al. Epidemiological trends in Merkel cell carcinoma in southern France: a registry-based study. $\mathrm{Br}$ J Dermatol 2017;176:1379-81.

3 Garbutcheon-Singh KB, Curchin DJ, McCormack CJ, et al. Trends in the incidence of Merkel cell carcinoma in Victoria, Australia, between 1986 and 2016. Australas J Dermatol 2020;61:e34-8.

4 Fondain M, Dereure O, Uhry Z, et al. Merkel cell carcinoma in France: a registries-based, comprehensive epidemiological survey. J Eur Acad Dermatol Venereol 2018;32:1292-6.

5 Lemos BD, Storer BE, lyer JG, et al. Pathologic nodal evaluation improves prognostic accuracy in Merkel cell carcinoma: analysis of 5823 cases as the basis of the first consensus staging system. J Am Acad Dermatol 2010;63:751-61.

6 Santamaria-Barria JA, Boland GM, Yeap BY, et al. Merkel cell carcinoma: 30-year experience from a single institution. Ann Surg Oncol 2013;20:1365-73.

7 Iyer JG, Blom A, Doumani R, et al. Response rates and durability of chemotherapy among 62 patients with metastatic Merkel cell carcinoma. Cancer Med 2016;5:2294-301.

8 Kaufman HL, Russell J, Hamid O, et al. Avelumab in patients with chemotherapy-refractory metastatic Merkel cell carcinoma: a multicentre, single-group, open-label, phase 2 trial. Lancet Oncol 2016;17:1374-85.

9 Feng $\mathrm{H}$, Shuda $\mathrm{M}$, Chang $\mathrm{Y}$, et al. Clonal integration of a polyomavirus in human Merkel cell carcinoma. Science 2008;319:1096-100.

10 Busam KJ, Jungbluth AA, Rekthman N, et al. Merkel cell polyomavirus expression in Merkel cell carcinomas and its absence in combined tumors and pulmonary neuroendocrine carcinomas. Am J Surg Pathol 2009;33:1378-85.

11 Schadendorf D, Nghiem P, Bhatia S, et al. Immune evasion mechanisms and immune checkpoint inhibition in advanced Merkel cell carcinoma. Oncoimmunology 2017;6:e1338237.
12 Wong SQ, Waldeck K, Vergara IA, et al. UV-Associated mutations underlie the etiology of MCV-Negative Merkel cell carcinomas. Cancer Res 2015;75:5228-34.

13 Knepper TC, Montesion M, Russell JS, et al. The genomic landscape of Merkel cell carcinoma and Clinicogenomic biomarkers of response to immune checkpoint inhibitor therapy. Clin Cancer Res 2019;25:5961-71.

14 Goh G, Walradt T, Markarov V, et al. Mutational landscape of MCPyV-positive and MCPyV-negative Merkel cell carcinomas with implications for immunotherapy. Oncotarget 2016;7:3403-15.

15 Miller NJ, Church CD, Fling SP, et al. Merkel cell polyomavirusspecific immune responses in patients with Merkel cell carcinoma receiving anti-PD-1 therapy. J Immunother Cancer 2018;6:131.

16 D'Angelo SP, Russell J, Lebbé C, et al. Efficacy and safety of first-line Avelumab treatment in patients with stage IV metastatic Merkel cell carcinoma: a Preplanned interim analysis of a clinical trial. JAMA Oncol 2018;4:e180077.

17 Nghiem P, Bhatia S, Lipson EJ, et al. Durable tumor regression and overall survival in patients with advanced Merkel cell carcinoma receiving pembrolizumab as first-line therapy. $J$ Clin Oncol 2019;37:693-702.

18 EMA. Summary Of Opinion - Initial Authorisation of Avelumab. Committee for Medicinal Products for Human Use 2017(EMA CHMP/426201/2017).

19 Walker JW, Lebbé C, Grignani G, et al. Efficacy and safety of avelumab treatment in patients with metastatic Merkel cell carcinoma: experience from a global expanded access program. $J$ Immunother Cancer 2020;8:e000313.

20 Pulitzer MP, Brannon AR, Berger MF, et al. Cutaneous squamous and neuroendocrine carcinoma: genetically and immunohistochemically different from Merkel cell carcinoma. Mod Pathol 2015;28:1023-32.

21 Calder KB, Smoller BR. New insights into Merkel cell carcinoma. Adv Anat Pathol 2010;17:155-61.

22 LMv V, Bertolli E, Korse C, et al. The clinical utility of neuron-specific enolase serum levels as a biomarker for Merkel cell carcinoma. Presented at ASCO 2019.

23 D'Angelo SP, Hunger M, Brohl AS, et al. Early objective response to avelumab treatment is associated with improved overall survival in patients with metastatic Merkel cell carcinoma. Cancer Immunol Immunother 2019;68:609-18.

24 D'Angelo SP, Bhatia S, Brohl AS, et al. Avelumab in patients with previously treated metastatic Merkel cell carcinoma: long-term data and biomarker analyses from the single-arm phase 2 javelin Merkel 200 trial. J Immunother Cancer 2020;8:e000674.

25 Sridharan V, Muralidhar V, Margalit DN, et al. Merkel cell carcinoma: a population analysis on survival. J Natl Compr Canc Netw 2016;14:1247-57.

26 Agelli M, Clegg LX. Epidemiology of primary Merkel cell carcinoma in the United States. J Am Acad Dermatol 2003;49:832-41.

27 Allen PJ, Bowne WB, Jaques DP, et al. Merkel cell carcinoma: prognosis and treatment of patients from a single institution. $J$ Clin Oncol 2005;23:2300-9.

28 Harms KL, Healy MA, Nghiem P, et al. Analysis of prognostic factors from 9387 Merkel cell carcinoma cases forms the basis for the new 8th edition AJCC staging system. Ann Surg Oncol 2016;23:3564-71.

29 Liang E, Brower JV, Rice SR, et al. Merkel cell carcinoma analysis of outcomes: a 30-year experience. PLoS One 2015;10:e0129476.

30 Tarantola TI, Vallow LA, Halyard MY, et al. Prognostic factors in Merkel cell carcinoma: analysis of 240 cases. J Am Acad Dermatol 2013;68:425-32.

31 Hui AC, Stillie AL, Seel M, et al. Merkel cell carcinoma: 27-year experience at the peter MacCallum cancer centre. Int J Radiat Oncol Biol Phys 2011;80:1430-5

32 Kaufman HL, Russell JS, Hamid O, et al. Updated efficacy of avelumab in patients with previously treated metastatic Merkel cell carcinoma after $\geq 1$ year of follow-up: JAVELIN Merkel 200, a phase 2 clinical trial. J Immunother Cancer 2018;6:7.

33 Vandeven N, Lewis CW, Makarov V, et al. Merkel cell carcinoma patients presenting without a primary lesion have elevated markers of immunity, higher tumor mutation burden, and improved survival. Clin Cancer Res 2018;24:963-71.

34 Tarantola TI, Vallow LA, Halyard MY, et al. Unknown primary Merke cell carcinoma: 23 new cases and a review. J Am Acad Dermatol 2013;68:433-40.

35 van Veenendaal LM, van Akkooi ACJ, Verhoef C, et al. Merkel cell carcinoma: clinical outcome and prognostic factors in 351 patients. $J$ Surg Oncol 2018;117:1768-75.

36 Hooiveld-Noeken JS, Fehrmann RSN, de Vries EGE, et al. Driving innovation for rare skin cancers: utilizing common tumours and machine learning to predict immune checkpoint inhibitor response. Immuno-Oncology Technology 2019;4:1-7. 